\title{
Agentes pedagógicos y su multiplicidad de roles
}

\author{
Alberto A. Piedrahita Ospina ${ }^{1,2}$, Julián Moreno Cadavid ${ }^{2}$, Néstor D. Duque \\ Méndez ${ }^{3}$ \\ ${ }^{1}$ Departamento de Educación y Ciencias Básicas - Instituto Tecnológico Metropolitano \\ Medellín, Colombia \\ ${ }^{2}$ Departmento de Ciencias de la Computación y la Decisión - Universidad Nacional de Colombia \\ Medellín, Colombia \\ ${ }^{3}$ Departamento de Informática y Computación - Universidad Nacional de Colombia \\ Manizales, Colombia \\ albertopiedrahita@itm.edu.co,jmoreno1@unal.edu.co
}

Resumen. Los agentes pedagógicos surgen como una alternativa, generalmente asociada a los sistemas tutoriales inteligentes, para proveer una interacción más "natural" con los estudiantes dentro de un ambiente virtual de aprendizaje. Para ello, tales agentes pueden llevar a cabo diversos roles dependiendo de su abordaje pedagógico, siendo el objetivo de este trabajo presentar una clasificación clara de los mismos, la cual parte de la descripción de sus características y las funciones que llevan a cabo. Por último, y a manera de ejemplo, se discriminan varios de agentes pedagógicos más referenciados en la literatura de acuerdo a tal clasificación.

Resumo. Os agentes pedagógicos surgem como uma alternativa, geralmente associada aos sistemas tutores inteligentes, para proporcionar uma interação mais "natural" com os alunos dentro de um ambiente virtual de aprendizagem. Para tanto, tais agentes podem exercer diferentes papéis dependendo de sua abordagem pedagógica, tendo como objetivo este artigo o apresentar uma classificação clara dos mesmos, a partir da descrição de suas características e das funções que desempenham. Finalmente, e a título de exemplo, vários dos agentes pedagógicos mais referenciados na literatura são discriminados de acordo com essa classificação.

\section{Introducción}

Una forma de proveer interactividad y dinamismo a los ambientes CAL (por sus siglas en inglés para Computer Assisted Learning) es a través de un Agente Pedagógico, los cuales se desarrollan para fines educativos con el fin de apoyar y asistir el proceso de enseñanza y aprendizaje (Frozza, da Silva, Lux, da Cruz, \& Borin, 2009).

Los agentes pedagógicos se refieren a agente inteligentes que tienen un rol pedagógico o educacional para facilitar o mejorar el aprendizaje (Jaques, 2004). Estos actúan como tutores virtuales, estudiantes virtuales o también como compañeros virtuales de aprendizaje, que tienen como propiedades fundamentales: autonomía, habilidad social, proactividad y persistencia. Estos agentes podrían tener la capacidad de aprender, son esencialmente cognitivos, pero pueden presentar características reactivas (Giraffa, 
VII Congresso Brasileiro de Informática na Educação (CBIE 2018)

Anais do XXIX Simpósio Brasileiro de Informática na Educação (SBIE 2018)

1999). De manera más reciente, (Kim, Thayne, \& Wei, 2017) mencionan el concepto de Agente Incorporado, que se refiere a un agente animado o un caractér digital embebido dentro de un sistema CAL para asistir a los estudiantes durante su proceso educativo.

El fundamento psicológico de un Agente Pedagógico se basa en el estudio de (Lester et al., 1997), quienes denominaron "efecto persona" al fenómeno que señala que la presencia de un carácter realista en un ambiente de aprendizaje interactivo, incluso que no sea expresivo, puede tener un efecto positivo en la percepción de los estudiantes durante su experiencia de aprendizaje. Posteriormente, otros estudios muestran evidencias de la efectividad que puede tener la incorporación de un agente pedagógico (Baylor, 2011), es decir, un agente gráfico presente en la educación mediada por computador, puesto que elude a ese comportamiento social natural que tienen las personas de comunicarse entre ellas, y aún más si se considera un ambiente social educativo, en el cual se presenta el proceso de enseñanza y aprendizaje.

Posteriormente, investigaciones han buscado ahondar en cuál es el diseño óptimo para un agente pedagógico (Rosenberg-Kima, Plant, Doerr, \& Baylor, 2010), donde se ha manifestado un predominio de los aspectos: gráfico, es decir el aspecto visual del agente; y comunicativo, que se refiere a las habilidades comunicativas del agente. En cuanto al aspecto visual, se debe considerar el realismo del agente y el ajuste de éste a la población objetivo, tal como, el género, la forma de vestir, la edad, la etnia, así como también, la forma de hablar, entre otros detalles que podrían permitir una mayor identificación entre el usuario y el agente. Algunas investigaciones han mencionado que, para cierto rango de edad, los agentes de género femenino han resultado más efectivos para estudiantes jóvenes, quizás porque asemejan a madres, y por ende podrían ser más persuasivos y motivacionales (Baylor, 2011). El segundo aspecto importante en el diseño de un agente pedagógico animado es la comunicación y despliegue de mensajes al usuario (Baylor, 2011), es importante en este aspecto considerar que la comunicación puede ser de varios tipos, mediante el envío de mensajes, el cual puede ser texto y mensajes de dialogo hablados, o quizás con un lenguaje no verbal que despliegue características, tales como, gestos, posturas, expresión facial, entre otros.

Otro aspecto importante por considerar es el rol del agente, es decir la función de éste durante el proceso educativo. De acuerdo con (C.-Y. Chou, Chan, \& Lin, 2003), y (Kim \& Baylor, 2006), existen dos grandes vertientes en cuanto al comportamiento: en primer lugar, el agente pedagógico autoritario, que se refiere a aquél agente tutor o profesor tradicional, que tiene el conocimiento formal, aquél que domina el conocimiento, aquél que es estricto y formal; en segundo lugar, el agente pedagógico no-autoritario también conocido como Sistema Acompañante de Aprendizaje o LCS (por sus siglas en inglés para Learning Companion System), el cual cumple roles más similares a un estudiante dentro de un proceso educativo, tales como, par tutor, tuteado o estudiante a enseñar, colaborador, competidor, creador de problemas, crítico o clon, donde cada uno de estos roles presentes en el proceso educativo de un estudiante real puede tener efectos psicológicos para su aprendizaje.

Debido a la diversidad de agentes pedagógicos en la comunidad científica, este artículo construye una taxonomía de agentes pedagógicos, discriminando dos rasgos, en primer lugar, el rol o la función que cumple, y por otro lado las características que presenta. 
VII Congresso Brasileiro de Informática na Educação (CBIE 2018)

Anais do XXIX Simpósio Brasileiro de Informática na Educação (SBIE 2018)

\section{Definición de roles y funciones}

De acuerdo con (C.-Y. Chou et al., 2003), existen dos grandes categorías para clasificar los agentes pedagógicos: autoritarios y no autoritarios. Los agentes pedagógicos autoritarios se presentan al estudiante, como expertos que no cometen errores en el dominio del conocimiento. Los agentes pedagógicos no autoritarios han sido denominados por los investigadores de diferente manera: "Learning Companion", "Colearner", "simulated student" y "artificial student", no obstante, a pesar de la diferencia en su nombre, todos se presentan al estudiante como poco expertos en el dominio del conocimiento y pueden cometer errores en el proceso de aprendizaje. Una amplia mayoría de estudios indican que los agentes pedagógicos no-autoritarios presentan ventajas en el proceso de aprendizaje (Hietala \& Niemirepo, 1998).

Tutor: El tutor (Sleeman \& Brown, 1982) ha sido el primer comportamiento, en el cual se evidencia el enfoque de agente pedagógico autoritario, donde el agente siempre tiene mayor conocimiento que el estudiante. El aprendiz recibe conocimiento únicamente del tutor, de acuerdo con comportamientos prestablecidos. Este enfoque se caracteriza además por presentar características adaptativas a mayor o menor medida, $\mathrm{y}$ dependiendo de la complejidad del sistema puede proporcionar retroalimentación al estudiante. En esta función, el conocimiento no se construye como una interacción bidireccional. Se presenta progresivamente el contenido al estudiante. No hay discusión e intercambio de puntos de vista entre el Agente Pedagógico y el estudiante.

Par Tutor (Peer Tutor): Algunos autores están de acuerdo en que la función cognitiva está sujeta a ambientes sociales lo cual implica que la interacción social con compañeros de aula influencia positivamente aspectos afectivos y cognitivos, óptimos para el aprendizaje. En este mismo sentido, (Schunk, 1987), plantea después de una revisión de la literatura, que la autoeficacia de los estudiantes se incrementa al estar junto a pares académicos con similar o más bajo desempeño, lo cual permite evaluar sus habilidades y expresarse con mayor confianza.

Colaborador (Collaborator): Cuando surgen dudas durante el proceso de aprendizaje de un estudiante, es natural que éste busque a un profesor, quien es la persona que representa la autoridad en el tema, sin embargo, el estudiante puede inclinarse por consultar a un compañero de clase en búsqueda de resolver sus dudas, y así evitar enfrentar al profesor con sus problemas (Chan \& Baskin, 1990). Este tipo de comportamiento indica que existe colaboración. Hietala \& Niemirepo (1998), señalan que la colaboración en un agente pedagógico está presente cuando esté apoya al estudiante durante la ejecución de actividades instruccionales activando con ello sus procesos cognitivos, adicionalmente, plantean los autores, un agente pedagógico colaborador no debe mostrarse ansioso, interrumpiendo constantemente al estudiante, debe progresivamente decrementando sus intervenciones hasta que el aprendiz logre realizar las tareas de manera autónoma.

Competidor (Competitor): Según (Chan \& Baskin, 1990), cuando se asignan las mismas actividades a dos estudiantes en un aula de clase pero separadas entre estos, tales como un taller de ejercicios matemáticos, es muy común que estos comiencen a comparar sus resultados, descubran sus errores y autocorrigen sus propias soluciones, por ende es posible indicar que existe competencia en ambos estudiantes. Un agente pedagógico competidor tendrá los mismos objetivos del estudiante real, presentando 
VII Congresso Brasileiro de Informática na Educação (CBIE 2018)

Anais do XXIX Simpósio Brasileiro de Informática na Educação (SBIE 2018)

siempre un umbral de referencia en cuanto al desempeño del estudiante. En un ambiente sin competencia, un estudiante real puede realizar los procedimientos propuestos por el profesor de manera cuidadosa y pausada, tomando los recursos a su disposición. Cuando se incorpora la competencia en las actividades, el estudiante se obliga a sí mismo a memorizar reglas y procedimientos, interiorizar rápidamente macrooperaciones para hallar rápidamente relaciones entre los conceptos.

Estudiante que enseñar (Tutee or Teachable): Chou \& Chan (1997) mencionan la posibilidad de que un tutor real interactúe con un aprendiz virtual (RT+VL), es decir un agente pedagógico que se comporte como un aprendiz falto de conocimiento y que comete errores, buscando en este proceso que el estudiante real mejore su aprendizaje por el simple hecho de enseñar. Este proceso ha sido denominado en algunos casos como modelo de aprendizaje por tutoría (learning by tutoring model) consiste en poner al estudiante en la posición de un tutor. De acuerdo con Biswas et al. (2001) los estudiantes que se preparan para enseñar obtienen un aprendizaje más profundo en el tema que pretenden enseñar, mediante una organización conceptual más clara, donde finalmente concluyen que aquellos estudiantes que enseñan a otros aprenden más que aquellos que son enseñados.

Provocador (Troublemaker): Aimeur \& Frasson (1996), proponen una nueva estrategia de aprendizaje aplicada a los agentes pedagógicos denominada aprendizaje por perturbación (learning by disturbing) en la cual el agente pedagógico es un compañero de aprendizaje que se comporta como problemático o alborotador, el cual en momentos da buenos consejos al aprendiz, pero también recomendaciones erradas. El objetivo de este comportamiento es provocar la reacción del estudiante para que proponga la solución correcta. También este rol de agente pedagógico puede esperar por la solución del estudiante y dar una sugerencia errada o un contra ejemplo al estudiante, para poner a dudar el estudiante. De acuerdo con los autores esta estrategia conduce a estudiantes argumentativos que defienden sus opiniones.

\section{Conclusiones}

Los agentes pedagógicos se han diversificado en una gran variedad de roles, siendo algunos: Tutor, Par Tutor, Aprendiz, Colaborador, Competidor y Provocador. El primero suele ser el más referenciado en la literatura científica y corresponde a un tipo de agente cuyo comportamiento implica una superioridad académica con el estudiante, tal como un docente o profesor real, el cual tiene como único objetivo mejorar el aprendizaje del estudiante. Desde una mirada más amplia de esto roles, puede decirse que los mismos corresponden a los modelos de aprendizaje heteroaprendizaje y coaprendizaje, los cuales varían de acuerdo con la fuente de enseñanza y el sumidero de aprendizaje, los cuales a su vez corresponden a la corriente pedagógica del conductismo y del constructivismo respectivamente.

\section{Referencias}

Aimeur, E., \& Frasson, C. (1996). Analyzing a new learning strategy according to different knowledge levels. Computers \& Education, 27(2), 115-127. https://doi.org/10.1016/0360-1315(96)00018-8 
VII Congresso Brasileiro de Informática na Educação (CBIE 2018)

Anais do XXIX Simpósio Brasileiro de Informática na Educação (SBIE 2018)

Baylor, A. L. (2011). The design of motivational agents and avatars. Educational Technology Research and Development, 59(2), 291-300.

Biswas, G., Katzlberger, T., Bransford, J., \& Schwartz, D. (2001). Extending Intelligent Learning Environments with Teachable Agents to Enhance Learning. En Artificial Intelligence in Education (pp. 389-397).

Chan, T.-W., \& Baskin, A. B. (1990). Learning Companion Systems, 26.

Chou, C.-Y., Chan, T.-W., \& Lin, C.-J. (2003). Redefining the learning companion: the past, present, and future of educational agents. Computers \& Education, 40(3), 255-269.

Chou, T. W., \& Chan, C. Y. (1997). Exploring the Design of Computer Supports for Reciprocal Tutoring. International Journal of Artificial Intelligence in Education, $8,1-24$.

Frozza, R., da Silva, A. K., Lux, B., da Cruz, M. E. K., \& Borin, M. (2009). Dóris 3D: Agente pedagógico baseado em emoções. En Brazilian Symposium on Computers in Education (Simpósio Brasileiro de Informática na Educação-SBIE) (Vol. 1).

Giraffa, L. M. M. (1999). Uma arquitetura de tutor utilizando estados mentais. Universidade Federal do Rio Grande do Sul, Porto Alegre.

Hietala, P., \& Niemirepo, T. (1998). The Competence of Learning Companion Agents. International Journal of Artificial Intelligence in Education (IJAIED), 9, 178-192.

Jaques, P. A. (2004). Using an animated pedagogical agent to interact affectively with the student. Universidade Federal do Rio Grande do Sul, Porto Alegre, Brazil.

Kim, Y., \& Baylor, A. L. (2006). Pedagogical agents as learning companions: The role of agent competency and type of interaction. Educational Technology Research and Development, 54(3), 223-243.

Kim, Y., Thayne, J., \& Wei, Q. (2017). An embodied agent helps anxious students in mathematics learning. Educational Technology Research and Development, 65(1), 219-235.

Lester, J. C., Converse, S. A., Kahler, S. E., Barlow, S. T., Stone, B. A., \& Bhogal, R. S. (1997). The persona effect: affective impact of animated pedagogical agents. En Proceedings of the ACM SIGCHI Conference on Human factors in computing systems (pp. 359-366). ACM.

Rosenberg-Kima, R. B., Plant, E. A., Doerr, C. E., \& Baylor, A. L. (2010). The influence of computer-based model's race and gender on female students' attitudes and beliefs towards engineering. Journal of Engineering Education, 99(1), 35-44.

Schunk, D. H. (1987). Peer models and children's behavioral change. Review of educational research, 57(2), 149-174.

Sleeman, D., \& Brown, J. S. (1982). Intelligent Tutoring Systems. London: Academic Press. Recuperado a partir de https://hal.archives-ouvertes.fr/hal-00702997 